\title{
Lattice QCD Equation of State for Nonvanishing Chemical Potential by Resumming Taylor Expansions
}

\author{
Sourav Mondal, ${ }^{1}$ Swagato Mukherjee $\odot,{ }^{2}$ and Prasad Hegde $\odot^{1, *}$ \\ ${ }^{1}$ Centre for High Energy Physics, Indian Institute of Science, Bangalore 560012, India \\ ${ }^{2}$ Physics Department, Brookhaven National Laboratory, Upton, New York 11973, USA
}

(Received 15 June 2021; accepted 9 December 2021; published 11 January 2022)

\begin{abstract}
Taylor expansion in powers of baryon chemical potential $\left(\mu_{B}\right)$ is an oft-used method in lattice QCD to compute QCD thermodynamics for $\mu_{B}>0$. Based only upon the few known lowest order Taylor coefficients, it is difficult to discern the range of $\mu_{B}$ where such an expansion around $\mu_{B}=0$ can be trusted. We introduce a resummation scheme for the Taylor expansion of the QCD equation of state in $\mu_{B}$ that is based on the $n$-point correlation functions of the conserved current $\left(D_{n}\right)$. The method resums the contributions of the first $N$ correlation function $D_{1}, \ldots, D_{N}$ to the Taylor expansion of the QCD partition function to all orders in $\mu_{B}$. We show that the resummed partition function is an approximation to the reweighted partition function at $\mu_{B} \neq 0$. We apply the proposed approach to high-statistics lattice QCD calculations using $2+1$ flavors of Highly Improved Staggered Quarks with physical quark masses on $32^{3} \times 8$ lattices and for temperatures $T \approx 145-176 \mathrm{MeV}$. We demonstrate that, as opposed to the Taylor expansion, the resummed version not only leads to improved convergence but also reflects the zeros of the resummed partition function and severity of the sign problem, leading to its eventual breakdown. We also provide a generalization of scheme to include resummation of powers of temperature and quark masses in addition to $\mu_{B}$, and show that the alternative expansion scheme of [S. Borsányi et al., Phys. Rev. Lett. 126, 232001 (2021).] is a special case of this generalized resummation.
\end{abstract}

DOI: 10.1103/PhysRevLett.128.022001

Introduction.-Lattice quantum chromodynamics (QCD) results for the QCD equation of state (EOS) plays a critical role in the dynamical modeling of heavy-ion collisions [1-4] and, thereby, in the experimental explorations of the QCD phase diagram in the $T-\mu_{B}$ plane. Because of the fermion sign problem it is difficult to carry out lattice QCD computations directly at $\mu_{B} \neq 0$. Despite some recent progress [5-10], direct lattice computations of the QCD EOS $\mu_{B} \neq 0$ with physical quark masses, fine lattice spacings, and large lattice volumes have remained elusive. Instead, the present state-of-the-art lattice QCD EOS at $\mu_{B}>0$ has been obtained using the Taylor expansion $[11,12]$ and the analytic continuation $[13,14]$ methods. In the Taylor expansion method one expands the pressure in powers of $\mu_{B}$ around $\mu_{B}=0$ and directly computes the Taylor coefficients at $\mu_{B}=0$. For the analytic continuation, one avoids the fermion sign problem using simulations at purely imaginary values $\mu_{B}$, fits these results with a power series in $\mu_{B}$ to determine the Taylor coefficients at $\mu_{B}=0$ and then provides the EOS at real $\mu_{B}>0$ based on these

Published by the American Physical Society under the terms of the Creative Commons Attribution 4.0 International license. Further distribution of this work must maintain attribution to the author(s) and the published article's title, journal citation, and DOI. Funded by SCOAP .
Taylor coefficients. On the other hand, it is well known that the applicability of the Taylor expansion as well as the analytic continuation should be limited by the zeros, nearest to $\mu_{B}=0$, of the partition function in the entire complex- $\mu_{B}$ plane [15-17]. In principle, it is possible to estimate the zeros of the partition function by re-expressing the power series in real or imaginary $\mu_{B}$ in terms of Padé approximants [12] or in a power series of the fugacity [18-20]. Armed, in reality, with only the few lowest order Taylor coefficients, this becomes a very difficult task and, in practice, one just restricts the EOS to $\left\{T, \mu_{B}\right\}$ that avoids any pathological nonmonotonicity in the truncated Taylor series $[11,14]$. Furthermore, these methods provide very little guidance on the severity of the fermion problem, i.e., how rapidly the phase of the partition function fluctuates as $\mu_{B}$ is increased. It is possible to determine the zeros of the partition function as well as its average phase by reweighting the fermion determinant to $\mu_{B} \neq 0$ [21-25]. However, due to the computational cost associated with exact evaluation of the fermion determinant, at present this method is restrained within coarse lattice spacings and small lattice volumes.

In this work, we introduce a method for the calculation of the lattice QCD EOS that genuinely resums the truncated Taylor series to all orders in $\mu_{B}$ and whose breakdown encodes the severity of the sign problem and zeros of the resummed partition function. 
The resummation method.-The Taylor expansion to $\mathcal{O}\left(\mu_{B}^{N}\right)$ of the excess pressure, $\Delta P\left(T, \mu_{B}\right) \equiv P\left(T, \mu_{B}\right)-$ $P(T, 0)$, is given by

$$
\frac{\Delta P_{N}^{E}}{T^{4}}=\sum_{n=1}^{N} \frac{\chi_{n}^{B}}{n !}\left(\frac{\mu_{B}}{T}\right)^{n}
$$

where the Taylor coefficients are defined as

$$
\chi_{n}^{B}(T)=\left.\frac{1}{V T^{3}} \frac{\partial^{n} \ln Z\left(T, \mu_{B}\right)}{\partial\left(\mu_{B} / T\right)^{n}}\right|_{\mu_{B}=0} .
$$

Here, the QCD partition function is denoted as $Z=\int e^{-S} \operatorname{det}[M] \mathcal{D} U, V$ is the spatial volume, $U$ is the $\mathrm{SU}(3)$ gauge fields, $S$ is the pure gauge action, and $M$ is the fermion matrix. Each $\chi_{n}^{B}$ consists of a sum of terms like $\left\langle D_{i}^{a} D_{j}^{b} \cdots D_{k}^{c}\right\rangle$ with $i \cdot a+j \cdot b+\cdots+k \cdot c=n[26,27]$, where

$$
D_{n}(T)=\bar{D}_{n} \cdot n !=\left.\frac{\partial^{n} \ln \operatorname{det}\left[M\left(T, \mu_{B}\right)\right]}{\partial\left(\mu_{B} / T\right)^{n}}\right|_{\mu_{B}=0},
$$

and the $\langle\cdot\rangle$ denotes the average over gauge field ensembles at $\mu_{B}=0$, i.e., $\langle O\rangle=\int O e^{-S} \operatorname{det}[M(T, 0)] \mathcal{D} U / Z$. The physical interpretation of $D_{n}$ is simple for the continuum theory: $D_{n}=\int d \mathbf{x}_{\mathbf{1}} \cdots d \mathbf{x}_{\mathbf{n}} J_{0}\left(\mathbf{x}_{\mathbf{1}}\right) \cdots J_{0}\left(\mathbf{x}_{\mathbf{n}}\right)$ is the integrated $n$-point correlation function of the 0 th component of the conserved (baryon) current $J_{0}(\mathbf{x})$ at a space-time point $\mathbf{x}$. Note that, due to $C P$ symmetry of QCD all $D_{n}$ for $n=$ odd(even) are purely imaginary(real) and only the $n=$ even terms contribute to Eq. (1). In practice, lattice QCD computations of the $\chi_{N}^{B}$ involve computations of all $D_{n}$ for $n \leq N$ as intermediate steps, and $\chi_{N}^{B}$ are obtained from combinations of $D_{n}$ and their powers.

Contributions of various combinations of $D_{n}$ to the few lowest order Taylor coefficients are sketched in Fig. 1.

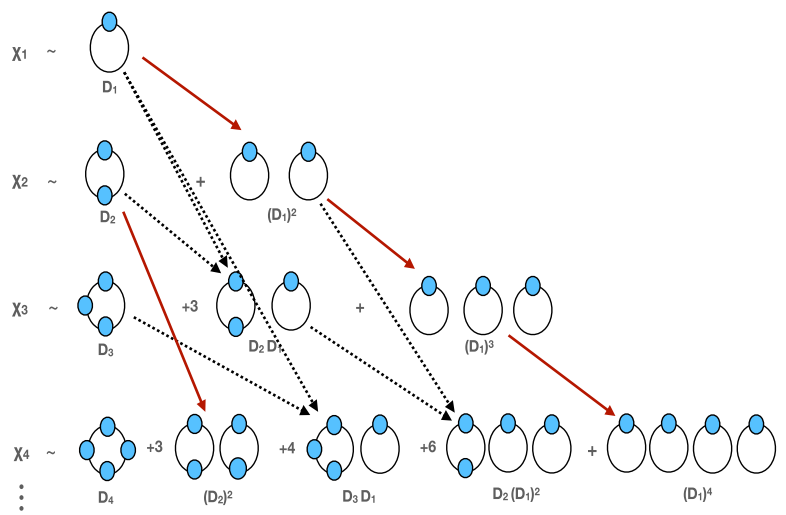

FIG. 1. Contributions of different $D_{n}$ to the $\chi_{n}^{B}$. Each blob represents insertion of the 0th component of the conserved current. Solid red and dotted black lines represent directly exponentiated and cross terms, respectively.
If one considers the factorials and the powers of $\mu_{B} / T$ associated with each $D_{n}$ in the sum of Eq. (1), it is not difficult to realize that all contributions of each $D_{n}$ to $\Delta P^{E}$ can be resummed into exponential forms. For example, contributions of $D_{1}^{n}$ from all $\chi_{n}^{B}$ in Eq. (1) can be resummed as $\exp \left[\bar{D}_{1}\left(\mu_{B} / T\right)\right]$. Similarly, contributions of all $D_{2}^{n}$ can be resummed as $\exp \left[\bar{D}_{2}\left(\mu_{B} / T\right)^{2}\right]$, and so on. Also it is easy to see that the contributions of the mixed terms like $D_{1}^{n} D_{2}^{m}$ arise from $\exp \left[\bar{D}_{1}\left(\mu_{B} / T\right)\right] \times \exp \left[\bar{D}_{2}\left(\mu_{B} / T\right)^{2}\right]$. Thus, it is possible to write down a resummed version of Eq. (1), viz.

$$
\frac{\Delta P_{N}^{R}}{T^{4}}=\frac{1}{T^{3} V} \ln \left\langle\exp \left[\sum_{n=1}^{N} \bar{D}_{n}\left(\frac{\mu_{B}}{T}\right)^{n}\right]\right\rangle,
$$

providing the EOS up to infinite orders in $\mu_{B}$. The $\Delta P_{N}^{R}$ can be considered as a $\mu_{B}$-dependent effective action obtained by resumming up to $N$-point correlation functions of the conserved current. Expansion of $\Delta P_{N}^{R}$ in powers of $\mu_{B} / T$ yields an infinite series in $\mu_{B} / T$, in addition to the truncated Taylor series: $\Delta P_{N}^{E}+\sum_{n>N}^{\infty}\left\langle\bar{D}_{1}^{i} \cdots \bar{D}_{N}^{j}\right\rangle\left(\mu_{B} / T\right)^{n}$, where $i, j=0, \ldots, N$ satisfying $1 \cdot i+\cdots+N j=n$. The Taylor expanded $\left(\mathcal{N}_{N}^{E}\right)$ and the resummed $\left(\mathcal{N}_{N}^{R}\right)$ net baryonnumber densities can be straightforwardly obtained as a single $\mu_{B}$ derivative of $\Delta P^{E}$ and $\Delta P^{R}$ in Eqs. (1) and (4), respectively.

The resummed version in Eq. (4) also highlights the connection between the Taylor expansion and the reweighting method. In the reweighting method $Z\left(T, \mu_{B}\right) / Z(T, 0)=$ $\left\langle\operatorname{det}\left[M\left(T, \mu_{B}\right)\right] / \operatorname{det}[M(T, 0)]\right\rangle$ can be calculated, if computationally feasible, by exactly evaluating the ratio of the fermion matrix determinants on the gauge fields generated at $\mu_{B}=0$. In more realistic lattice calculations with large volumes, exact evaluations of the determinant ratios might not be computationally feasible and one may consider evaluating $\operatorname{det}\left[M\left(T, \mu_{B}\right)\right]$ within some approximation scheme to obtain approximate partition function $Z_{N}^{R}\left(T, \mu_{B}\right) \approx Z\left(T, \mu_{B}\right)$. Following the spirit of the Taylor expansion, one such approximation scheme can be expansion of $\operatorname{det}\left[M\left(T, \mu_{B}\right)\right]$ in powers of $\mu_{B} / T$. Keeping in mind $\operatorname{det}[M]=\exp [\operatorname{Tr} \ln M]$ and Eq. (3), one can immediately recognize

$$
\frac{Z_{N}^{R}\left(T, \mu_{B}\right)}{Z(T, 0)}=\left\langle\exp \left[\sum_{n=1}^{N} \bar{D}_{n}\left(\frac{\mu_{B}}{T}\right)^{n}\right]\right\rangle .
$$

Since $C P$ symmetry dictates that the even(odd) $D_{n}$ are purely real(imaginary) and the partition function must be real, a measure of the severity of the sign problem is given by the average phase factor for $Z_{N}^{R}$ (with $\mu_{B}$ real),

$$
\left\langle\cos \Theta_{N}^{R}\right\rangle=\left\langle\cos \left(\sum_{n=1}^{N / 2} \operatorname{Im}\left[\bar{D}_{2 n-1}\right]\left(\frac{\mu_{B}}{T}\right)^{2 n-1}\right)\right\rangle .
$$


An expansion of $\left\langle\cos \Theta_{N}^{R}\right\rangle$ in $\mu_{B} / T$ leads to the Taylor expanded measure of the average phase of the partition function $[23,26]$, which we will denote by $\Theta_{N}^{E}$. As the sign problem becomes more severe the average phase $\left\langle\cos \Theta_{N}^{R}\right\rangle \approx 0$ and resummed results will also show signs of breakdown. Furthermore, although $\Delta P_{N}^{E}$ can be evaluated for any complex value of $\mu_{B}, \Delta P_{N}^{R}$ becomes undefined when $\operatorname{Re}\left[Z_{N}^{R}\right] \leq 0$ for a given $N$ and statistics, leading to a natural breakdown of the resummed results. The location of the zeros of $Z_{N}^{R}$ in the complex- $\mu_{B}$ plane will indicate the $\mu_{B}$ region where such resummation can be applicable. Obviously, for any given $N$ the region of applicability of $\Delta P_{N}^{E}$ cannot exceed the same for $\Delta P_{N}^{R}$.

Lattice $Q C D$ computations.-For this work, we used the data for $\chi_{n}^{B}$ and $D_{n}$ generated by the HotQCD Collaboration for calculations of the QCD EOS [11] and the chiral crossover temperature [28] at $\mu_{B}>0$ using the Taylor expansion method. The HotQCD ensembles were generated with $2+1$ flavors of Highly Improved Staggered Quarks and the tree-level improved Symanzik gauge action [29-31]. Bare quark masses were chosen to reproduce, within a few percent, the physical value of the kaon mass and a pseudo-Goldstone pion mass of $138 \mathrm{MeV}$ in the continuum limit at $T=\mu_{B}=0$ and the lattice spacing were calibrated against the physical value of the kaon decay constant [32]. We present lattice QCD results from a single lattice size $32^{3} \times 8$ and for 6 temperatures $T=145,151$, 157, 166, 171, $176 \mathrm{MeV}$. About 475, 520, 716, 522, 232, and $152 \mathrm{~K}$ gauge field configurations were used to measure $D_{n}$ at these temperatures, respectively. The gauge field configurations were separated by 10 Rational Hybrid Monte Carlo trajectories of unit length. The $D_{n}$ were calculated within the formalism adopted in Refs. [11,28], i.e., using the exponential- $\mu$ formalism [33] for $n \leq 4$ and the linear- $\mu$ formalism [34,35] for $n>4$. The expressions for $D_{n}$ in terms of the traces involving the inverse of the staggered fermion matrix and its $\mu_{B}$ derivatives are well known [26,36]. Each trace was calculated stochastically for each configuration by employing 2000 random Gaussian volume sources for the trace $D_{1}$ and 500 random sources for the rest [36].

Results.-To demonstrate the superiority of the resummation method over the Taylor expansion, we chose the temperature where we had the largest statistics, i.e., $T=157 \mathrm{MeV}$, which is also closest to the QCD crossover temperature [28]. In Fig. 2, we compare $\Delta P_{N}^{E}$ with $\Delta P_{N}^{R}$ (top) and $\mathcal{N}_{N}^{E}$ with $\mathcal{N}_{N}^{R}$ for different orders $N$. Comparisons are shown both for real as well as imaginary values of $\mu_{B}$, corresponding to positive and negative values of $\left(\mu_{B} / T\right)^{2}$, respectively. The $\Delta P_{N}^{R}$ and $\mathcal{N}_{N}^{R}$ show very good convergence between different orders $N=2,4,6,8$. The Taylorexpanded results seem to approach their respective resummed results as contributions from higher orders in $\mu_{B}$ are included; however, the convergence of the Taylor-expanded


FIG. 2. Comparisons between the Taylor expanded and resummed results for different orders for the excess pressure (top) and net baryon-number density (bottom) at $T=157 \mathrm{MeV}$. Results for real and imaginary $\mu_{B} / T$ are plotted on the positive and negative $x$ axis, respectively.

results is slow due to the alternating signs of the higher order $\chi_{n}^{B}$ near the QCD crossover [11-13]. The resummation method overcomes this problem by including contributions from all orders in $\mu_{B}$ and shows markedly improved convergence.

We checked that such a breakdown is not a mere statistical issue by repeating the calculations using only parts of the gauge configurations available at this temperature. Similar breakdown for $\mu_{B} / T \gtrsim 1.5$ was also observed in Refs. $[12,37,38]$ when the EOS was reconstructed from the Padé approximants of the Taylor series in $\mu_{B}$. While Padé-based continuations of the QCD crossover temperature from imaginary values $\mu_{B}$ did not encounter such breakdowns $[39,40]$, the same in the case of the EOS seemed to break down due to singularities in the complex$\mu_{B}$ plane [41].

To investigate the origin of this breakdown, we computed the average phase as a function of real $\mu_{B}$, cf. Eq. (6). The results are shown in Fig. 3 (top). Also, $\left\langle\cos \Theta_{N}^{R}\right\rangle \approx 0$ for $\mu_{B} / T \gtrsim 1.5$, which shows that the sign problem is uncontrollably severe where the EOS calculations broke down. The resummation method thus faithfully captures the severity of the sign problem, as opposed to the Taylor expansion. The phase factor cannot be calculated exactly within the Taylor series approach. Its Taylor series expansion too converges very slowly, as the bands plotted in Fig. 3 (top) show. Further, we searched for the zeros of resummed partition function, cf. Eq. (5), in the complex $\mu_{B}$ plane. We solved for $Z_{N}^{R}=0$ using the Newton-Raphson 



FIG. 3. (Top) The average phase factor $\left\langle\cos \Theta_{N}^{R}\right\rangle$ as a function of $\mu_{B} / T$. The bands are the Taylor series expansions of the phase factor to different orders. (Bottom) Zeros of $Z_{N}^{R}$ in the complex$\mu_{B}$ plane. Only roots in the first quadrant are shown since the distribution is symmetric in the four quadrants. Both top and bottom plots are for $T=157 \mathrm{MeV}$.

algorithm with initial guesses chosen from a uniform distribution over a grid $0 \leq\left\{\operatorname{Re}\left(\mu_{B} / T\right), \operatorname{Im}\left(\mu_{B} / T\right)\right\} \leq$ 2.5. The results are shown in Fig. 3 (bottom). The zeros of $Z_{6}^{R}$ and $Z_{8}^{R}$ are more or less consistent with each other and appears only for $\left|\mu_{B} / T\right| \gtrsim 1.5$. The exact nature of the singularity responsible for breakdown of the resummation method is certainly of great interest, i.e., whether it is associated with the Yang-Lee edge singularity of the QCD chiral transition $[15,17]$ or the QCD critical point and approaches the real axis $[12,21,22,37,38]$, etc. This will need detailed quantitative studies involving careful finitevolume scaling analyses using more sophisticated techniques $[18,20,42]$ and is beyond the scope of the present work. But our results demonstrate that the breakdown of the resummation method reflects the associated singularities of the partition function, at least qualitatively.

Finally, in Fig. 4 we summarize results for all $T=$ $145-176 \mathrm{MeV}$ by showing comparisons between $\Delta P_{6}^{R}$ and $\mathcal{N}_{6}^{R}$ with the corresponding $\Delta P_{6}^{E}$ and $\mathcal{N}_{6}^{E}$. As in the case of $T=157 \mathrm{MeV}, \Delta P^{R}$ and $\mathcal{N}^{R}$ show improved convergence over $\Delta P^{E}$ and $\mathcal{N}^{E}$ at all temperatures. Again, in contrast to the Taylor expansion the resummation method shows signs of breakdown for $\mu_{B} \gtrsim 200-250 \mathrm{MeV}$, depending on the temperature. As before, we checked that in all cases, these breakdowns reflect the severity of the sign problem and the singularities of the partition function in the complex$\mu_{B}$ plane.
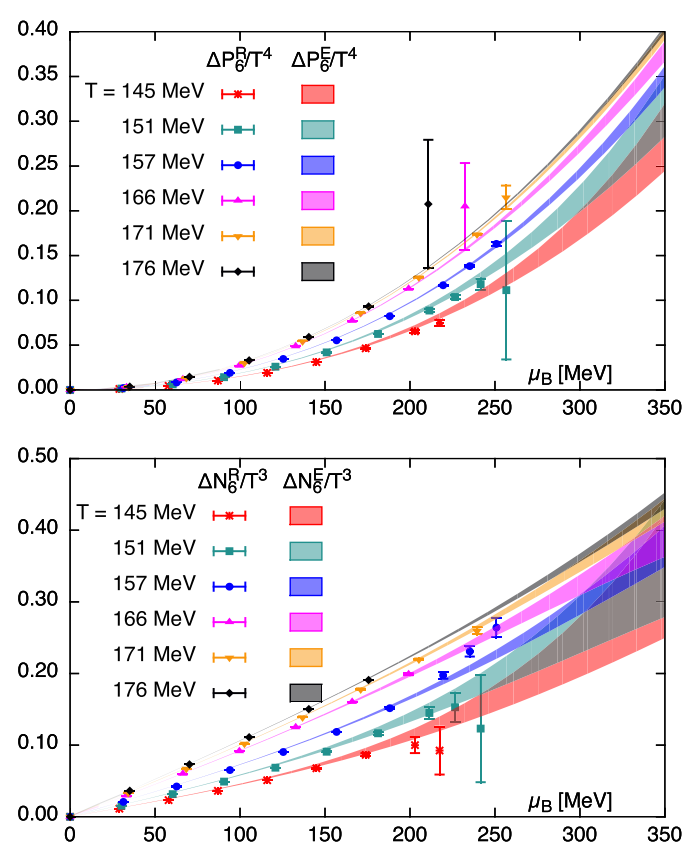

FIG. 4. Comparisons between the excess pressure (top) and the net baryon-number density (bottom) obtained the sixth order resummation $\left(\Delta P_{6}^{R}\right.$ and $\left.\mathcal{N}_{6}^{R}\right)$ and Taylor expansion $\left(\Delta P_{6}^{E}\right.$ and $\mathcal{N}_{6}^{E}$ ) methods for all six temperatures that were considered in this work.

Generalization to multiparameter and joint expansion in $T, \mu_{B}$.- Since our resummation scheme is equivalent to an approximate reweighting method, for large values of $\mu_{B}$ the results obtained using this method are subjected to the socalled overlap problem. The distribution of the gauge configurations at $\mu_{B}=0$ might be drastically different from that at large $\mu_{B}$, leading to smaller overlap between the two gauge field distributions and inaccurate results with increasing $\mu_{B}$. A hint of such an overlap problem may be seen in Fig. 2, where the resummed results for $\operatorname{Im}\left(\mu_{B}\right)$ not only deviate from the corresponding Taylor expansion results but also from the direct lattice QCD computations at $\operatorname{Im}\left(\mu_{B}\right)$ [14]. To mitigate such overlap problems, we propose a generalized resummation scheme that is akin to multiparameter reweighting [21-24] in the bare gauge coupling, $\Delta \beta=\beta-\beta_{0}$, and quark mass, $\Delta m=m-m_{0}$. Our resummation scheme also can be extended to obtain $Z_{N}^{R}\left(T, \mu_{B}\right)$ starting from a different temperature $T_{0}\left(\beta_{0}\right)$ and bare quark mass $m_{0}$,

$$
\frac{Z_{N}^{R}\left(T, \mu_{B}\right)}{Z\left(T_{0}, 0\right)}=\left\langle e^{-S_{G} \Delta \beta+\sum_{i+j=1}^{N} \bar{G}_{i j}\left(\frac{\mu_{B}}{T}\right)^{i}\left(\frac{\Delta m}{T}\right)^{j}}\right\rangle
$$

where the expectation value is taken over gauge fields associated with $\left\{\beta_{0}, m_{0}, 0\right\}$. Here, $S_{G}$ is the pure gauge action and 


$$
\overline{\mathcal{G}}_{i j}\left(\beta_{0}, m_{0}\right)=\left.\frac{\partial^{i} \partial^{j} \ln \operatorname{det}\left[M\left(m, \mu_{B}\right)\right]}{i ! j ! \partial\left(\mu_{B} / T\right)^{i} \partial(m / T)^{j}}\right|_{\left(m_{0}, 0\right)} .
$$

Note, $\overline{\mathcal{G}}_{i 0}=\bar{D}_{i}$ [Eq. (3)], $\overline{\mathcal{G}}_{0 j}$ are the chiral condensate and higher order chiral susceptibilities, and general $\overline{\mathcal{G}}_{i j}$ are $\mu_{B}$ derivatives of various chiral observables $[26,36,43,44]$. This generalization can possibly mitigate the overlap problem that one might encounter while resumming only in $\mu_{B}$. Further, a systematic expansion of the logarithm of Eq. (7) in powers of $\Delta \beta, \Delta m$, and $\mu_{B}$ yields the expansion of the pressure difference, $P\left(T, \mu_{B}\right) / T^{4}-P\left(T_{0}, \mu_{B}\right) / T_{0}^{4}$, in powers of $\Delta T=T-T_{0}$ and $\mu_{B}$; particular choice of $T_{0}\left(\mu_{B}\right)$ defined by a line of constant physics in the $T-\mu_{B}$ plane reproduces the expansion scheme used in Ref. [45] (see Supplemental Material [46]). Thus, our method also generalizes the alternative expansion scheme of Ref. [45] by resumming up to $N$-point baryon-current correlations to all orders in $\mu_{B}$ and $\Delta T$.

Conclusions. - We have introduced a new method to compute lattice QCD EOS by resumming contributions of up to $N$-point baryon-current correlations to all orders in $\mu_{B}$. When expanded in powers of $\mu_{B}$ this resummed partition function exactly reproduces the Taylor expansion up to $\mathcal{O}\left(\mu_{B}^{N}\right)$, plus an infinite series in $\mu_{B}$ capturing all possible contributions involving only the $n \leq N$-point baryon-current correlations. This resummation method also amounts to an approximate reweighting method, thereby bridging two traditional lattice QCD techniques for $\mu_{B} \neq 0$. With illustrative high-statistics lattice QCD computations we have demonstrated that the resummation method shows improved convergence over the Taylor expansion method. The method also faithfully captures the severity of the sign problem as well as reflects the singularities in the complex$\mu_{B}$ plane that are responsible for its eventual breakdown. Thus the resummation method not only provides a more convergent lattice QCD EOS but also a more reliable one by enabling us to judge its validity with increasing $\mu_{B}$. Although the resummation method is more general and powerful than the Taylor expansion, computationally it is somewhat simpler. The resummation method relies on the computations of $D_{n}$ which come as an intermediate step in the computations of the Taylor coefficients. Comparison with the resummed results and the direct lattice QCD simulations for purely imaginary $\mu_{B}$ will help us to decide up to what values $\operatorname{Im}\left(\mu_{B}\right)$ an analytic continuation using only the power series of $\mu_{B}$ is justified and whether Padétype analytic continuations [39-41] are necessary to avoid singularities in the complex- $\mu_{B}$ plane. We have also introduced a generalized multiparameter version of the resummation, Eq. (7), and shown that the method of Ref. [45] is a special case of this-Taylor expansion of Eq. (7) in $T$ and $\mu_{B}$ along a specific line in the $T-\mu_{B}$ plane. While the success of the method of Ref. [45] indicates that our generalized multiparameter resummation will mitigate the overlap problem to a large extent, the QCD equation of state presented in this work was not obtained using the generalized method and might suffer from the overlap problem.

We are indebted to members of the HotQCD Collaboration for letting us reuse the data they had generated for the Taylor expansion calculations as well as for several valuable discussions. This material is based upon work supported by The U.S. Department of Energy, Office of Science, Office of Nuclear Physics through the Contract No. DE-SC0012704; The U.S. Department of Energy, Office of Science, Office of Nuclear Physics and Office of Advanced Scientific Computing Research, within the framework of Scientific Discovery through Advance Computing (SciDAC) award Computing the Properties of Matter with Leadership Computing Resources; The U.S. Department of Energy, Office of Science, Office of Nuclear Physics, within the framework of the Beam Energy Scan Theory (BEST) Topical Collaboration. The Early Career Research Award of the Science and Engineering Research Board (SERB) of the Government of India; and The Institute Postdoc Fellowships of the Indian Institute of Science, Bangalore. This research used awards of computer time provided by the INCITE and ALCC programs at Oak Ridge Leadership Computing Facility, a DOE Office of Science User Facility operated under Contract No. DE-AC05-00OR22725. This work also made use of the clusters and data storage facilities located at Bielefeld University, Germany.

*prasadhegde@iisc.ac.in

[1] J. E. Bernhard, J. S. Moreland, S. A. Bass, J. Liu, and U. Heinz, Phys. Rev. C 94, 024907 (2016).

[2] P. Parotto, M. Bluhm, D. Mroczek, M. Nahrgang, J. Noronha-Hostler, K. Rajagopal, C. Ratti, T. Schäfer, and M. Stephanov, Phys. Rev. C 101, 034901 (2020).

[3] A. Monnai, B. Schenke, and C. Shen, Phys. Rev. C 100, 024907 (2019).

[4] D. Everett et al. (JETSCAPE Collaboration), Phys. Rev. C 103, 054904 (2021).

[5] M. Cristoforetti, F. Di Renzo, and L. Scorzato (Aurora Science Collaboration), Phys. Rev. D 86, 074506 (2012).

[6] D. Sexty, Phys. Lett. B 729, 108 (2014).

[7] M. Fukuma, N. Matsumoto, and N. Umeda, arXiv:1912 13303.

[8] G. Aarts, Proc. Sci., LAT2009 (2009) 024.

[9] G. Aarts, L. Bongiovanni, E. Seiler, D. Sexty, and I.-O. Stamatescu, Eur. Phys. J. A 49, 89 (2013).

[10] Z. Fodor, S. D. Katz, D. Sexty, and C. Török, Phys. Rev. D 92, 094516 (2015).

[11] A. Bazavov et al., Phys. Rev. D 95, 054504 (2017).

[12] S. Datta, R. V. Gavai, and S. Gupta, Phys. Rev. D 95, 054512 (2017).

[13] S. Borsanyi, Z. Fodor, J. N. Guenther, S. K. Katz, K. K. Szabo, A. Pasztor, I. Portillo, and C. Ratti, J. High Energy Phys. 10 (2018) 205. 
[14] J. N. Guenther, R. Bellwied, S. Borsanyi, Z. Fodor, S. D. Katz, A. Pasztor, C. Ratti, and K. K. Szabó, Nucl. Phys. A967, 720 (2017).

[15] M. A. Stephanov, Phys. Rev. D 73, 094508 (2006).

[16] G. A. Almási, B. Friman, K. Morita, and K. Redlich, Phys. Lett. B 793, 19 (2019).

[17] S. Mukherjee and V. Skokov, Phys. Rev. D 103, L071501 (2021).

[18] I. Barbour and A. Bell, Nucl. Phys. B372, 385 (1992).

[19] M. Giordano and A. Pásztor, Phys. Rev. D 99, 114510 (2019).

[20] M. Giordano, K. Kapas, S. D. Katz, D. Nogradi, and A. Pasztor, Phys. Rev. D 101, 074511 (2020).

[21] Z. Fodor and S. Katz, Phys. Lett. B 534, 87 (2002).

[22] Z. Fodor and S. Katz, J. High Energy Phys. 04 (2004) 050.

[23] S. Ejiri, Phys. Rev. D 69, 094506 (2004).

[24] H. Saito, S. Ejiri, S. Aoki, K. Kanaya, Y. Nakagawa, H. Ohno, K. Okuno, and T. Umeda, Phys. Rev. D 89, 034507 (2014).

[25] M. Giordano, K. Kapas, S. D. Katz, D. Nogradi, and A. Pasztor, J. High Energy Phys. 05 (2020) 088.

[26] C. Allton, M. Doring, S. Ejiri, S. Hands, O. Kaczmarek, F. Karsch, E. Laermann, and K. Redlich, Phys. Rev. D 71, 054508 (2005).

[27] R. V. Gavai and S. Gupta, Phys. Rev. D 68, 034506 (2003).

[28] A. Bazavov et al. (HotQCD Collaboration), Phys. Lett. B 795, 15 (2019).

[29] A. Bazavov et al., Phys. Rev. D 85, 054503 (2012).

[30] A. Bazavov et al. (HotQCD Collaboration), Phys. Rev. D 86, 034509 (2012).

[31] A. Bazavov et al. (HotQCD Collaboration), Phys. Rev. D 90, 094503 (2014).

[32] A. Bazavov et al., Phys. Rev. D 100, 094510 (2019).

[33] P. Hasenfratz and F. Karsch, Phys. Lett. 125B, 308 (1983).
[34] R. V. Gavai and S. Sharma, Phys. Rev. D 85, 054508 (2012).

[35] R. V. Gavai and S. Sharma, Phys. Lett. B 749, 8 (2015).

[36] P. Steinbrecher, The QCD crossover up to $O\left(\mu_{B}^{6}\right)$ from Lattice QCD, Ph.D. thesis, University of Bielefeld (main), 2018, https://pub.uni-bielefeld.de/download/2919977/ 2920377/qcd_crossover.pdf.

[37] S. Gupta, N. Karthik, and P. Majumdar, Phys. Rev. D 90, 034001 (2014).

[38] R. V. Gavai and S. Gupta, Phys. Rev. D 78, 114503 (2008).

[39] P. Cea, L. Cosmai, M. D’Elia, A. Papa, and F. Sanfilippo, Phys. Rev. D 85, 094512 (2012).

[40] A. Pásztor, Z. Szép, and G. Markó, Phys. Rev. D 103, 034511 (2021).

[41] C. Schmidt, J. Goswami, G. Nicotra, F. Ziesché, P. Dimopoulos, F. Di Renzo, S. Singh, and K. Zambello, Acta Phys. Pol. B Proc. Suppl. 14, 241 (2021).

[42] M. Wakayama, V. G. Bornyakov, D. L. Boyda, V. A. Goy, H. Iida, A. V. Molochkov, A. Nakamura, and V. I. Zakharov, Phys. Lett. B 793, 227 (2019).

[43] A. Bazavov et al. (HotQCD Collaboration), Phys. Lett. B 795, 15 (2019).

[44] S. Gupta and R. Ray, Phys. Rev. D 70, 114015 (2004).

[45] S. Borsányi, Z. Fodor, J. N. Guenther, R. Kara, S. D. Katz, P. Parotto, A. Pásztor, C. Ratti, and K. K. Szabó, Phys. Rev. Lett. 126, 232001 (2021).

[46] See Supplemental Material at http://link.aps.org/ supplemental/10.1103/PhysRevLett.128.022001 for a generalization of the method presented here to resummation in both $\mu_{B}$ and $T$. The generalized method resums the alternative expansion scheme presented in S. Borsányi et al. [45] to all orders in $\mu_{B}$ and $T$ and is equivalent to multi-parameter reweighting in $\mu_{B}$ and $T$. 